\title{
Perceptual memory over very short interstimulus intervals
}

\author{
DONALD LAMING \\ University of Cambridge, Cambridge, England \\ and \\ DARYL WIGHTMAN \\ Price Waterhouse and Company, Sydney, N.S.W., Australia \\ (Charles W. Eriksen, Sponsor)
}

\begin{abstract}
This experiment on discrimination between the length of two lines shows how the geometric range of interstimulus intervals (1 to $64 \mathrm{sec}$ ) examined by Laming and Scheiwiller (1985) might be extended down to small fractions of a second. Increasing the geometric range over which comparable measures of discriminability are obtained is likely to provide useful information about the nature of the underlying memory process.
\end{abstract}

This experiment on discrimination between the lengths of two briefly presented lines provides a comment on the work of Laming and Scheiwiller (1985). Laming and Scheiwiller reviewed five contemporary models that purported to describe the way in which discriminability decreased as the temporal interval between the presentations of two stimuli increased. The five models were compared with three existing and one fresh experiment on discrimination between two tones of different frequencies or two lines of different lengths. The results of that comparison were disappointing for the following reason:

Each experiment explored an empirical relation, as shown in Figure 1, between $d^{\prime}$ or inverse threshold, as the measure of discriminability, and the lapse of time between the two stimulus presentations. Each model incorporated a free parameter to accommodate the otherwise unspecified difficulty of the discrimination; this enabled the model's predictions to be aligned vertically with the experimental data. Each model also incorporated a parameter (e.g., an unspecified rate of exponential decay) that rescaled the time axis, allowing each model's predictions to be expanded or contracted laterally to achieve the best match between the gradient of the predictions and the trend of the data points. So the possibility of experimental discrimination between the different models depended entirely on differences in curvature between their respective predictions. Accurate assessment of curvilinear trends in experimental data requires a wide range in the independent variable. In this present case, it is the geometric range that matters because the free parameter rescaling the time

The experiment reported here was carried out by the second author with the assistance of a grant from the Charles Grindley Fund. Correspondence may be addressed to Donald Laming, University of Cambridge, Department of Experimental Psychology, Downing Street, Cambridge, England CB2 3EB. axis means that a range of retention intervals from 10 to $600 \mathrm{sec}$ is equivalent, under adjustment of that parameter, to a range from 1 to $60 \mathrm{sec}$. That particular range, common to all the experiments examined by Laming and Scheiwiller (1985), proved insufficient to discriminate between the different models of interest.

There are obvious difficulties in increasing the range of retention intervals much above $60 \mathrm{sec}$, but improved experimental discrimination could be achieved by extending the range downward toward 0 sec. Laming and Scheiwiller (1985) attempted this in a second experiment but encountered a different difficulty: for one of their two subjects, discrimination actually got worse as the retention interval was reduced below $0.25 \mathrm{sec}$. Such a loss of

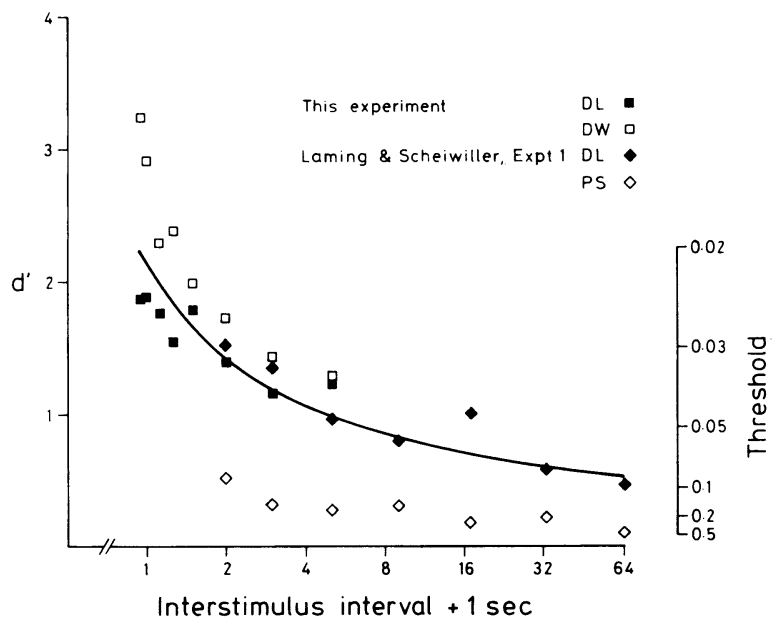

Figure 1. Discriminability (d'; squares) as a function of interstimulus interval, together with comparable data (inverse thresholds; diamonds) from Loming and Scheiwiller (1985, Experiment 1), compared with Equation 1. 
discriminability between two closely successive stimuli has previously been reported in the auditory modality by Tanner (1961) and Sorkin (1966).

In a series of preliminary experiments, we identified several factors that might interact with the discrimination of the length of two lines presented in very close succession. We presented our lines for $60 \mathrm{msec}$ each, instead of Laming and Scheiwiller's (1985) $500 \mathrm{msec}$, because Taylor and Smith (1975) reported that loss of discriminability in the auditory modality could be demonstrated only with briefly presented stimuli.

1. The configuration of the two lines. Since we intended the subject to discriminate the two lines specifically on the basis of their lengths, using no extraneous point of reference that might survive the retention interval, the lines were presented in random locations on the video display unit. As a consequence, they sometimes overlapped horizontally, one below the other. Greater overlap made the lengths easier to discriminate, especially at short intertrial intervals.

2. Fixation. When the lines were separated sufficiently to preclude overlap, being often presented at opposite sides of the screen, insufficient time to change fixation from the first line to the second made discrimination more difficult at short intertrial intervals.

3. Masking. We also tried physically superposing the lines by a random proportion, $50 \%-80 \%$, of their length. At zero intertrial interval (with the onset of the second line immediately following the offset of the first), the discrimination proved difficult, though still better than chance. But there was one session in our preliminary experiment when, by a programming error, that part of the first line that underlay the second was entirely omitted. Curiously, there was no demonstrable difference in discriminability.

In an attempt to evade all these complications, we chose the stimulus configuration shown in Figure 2.

\section{METHOD}

\section{Stimuli}

Green horizontal lines were presented against a dark background on a 12-in. video display unit (VDU) with P31 phosphor. The stimulus luminance was $430 \mathrm{~cd} / \mathrm{m}^{2}$, and the background luminance was $70 \mathrm{~cd} / \mathrm{m}^{2}$. Viewed from a distance of $60 \mathrm{~cm}$, the lines were $0.052^{\circ}$ thick and $1.3^{\circ}$ to $5.2^{\circ}$ long. Line length varied at random from trial to trial to prevent the subject using the length seen on a previous trial as a basis for judgment. This precaution was essential; Burbeck (1987) has found one subject, with unlimited inspection time, to perform at least as well (threshold about $2 \%$ ) when one length only was presented for comparison with a remembered standard (method of single stimuli) as when two lengths were presented simultaneously on each trial. Likewise, to minimize any contribution from contextual cues such as the frame of the VDU screen, the stimulus configuration (Figure 2) was entirely contained within an area $16.6^{\circ} \times 4.6^{\circ}$ centered within a screen of angular size $24.0^{\circ} \times 19.7^{\circ}$.

\section{Procedure}

The VDU was driven by a BBC microcomputer with a $50-\mathrm{Hz}$ refresh rate. Each trial began with the $0.2^{\circ}$ vertical marker, coterminous with an auditory warning signal. One second later, the first (left-hand) line was presented for a nominal $60 \mathrm{msec}$, and, after the chosen interstimulus

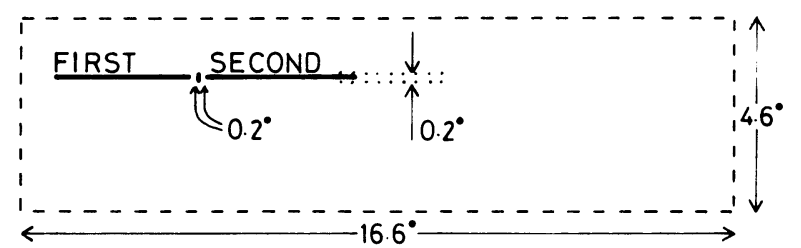

Figure 2. Configuration of the stimuli.

interval (ISI), the second line was presented for the same duration. One line was $8 \%$ longer than the other; this difference had been found, in the preliminary experiments, to yield about $75 \%$ correct responses. After each pair of stimuli, the subject expressed his confidence that the second (right-hand) line had been the longer on a 6-point scale, after the manner of Swets, Tanner, and Birdsall (1961, p. 326). Knowledge of results was given immediately following each response.

\section{Design}

Each subject contributed 4,480 trials divided between five 2-h sessions. Within each session, the trials were divided equally between all combinations of (1) two stimulus orders, longer, shorter and shorter, longer; (2) eight ISIs, true simultaneity (i.e., $-60 \mathrm{msec}$ ), 0 (immediate succession), $120,260,500,1,000,2,000$, and 4,000 msec, measured from offset of the first stimulus to onset of the second; and (3) seven standard lengths, $1.3^{\circ}$ to $5.2^{\circ}$ in steps of $0.65^{\circ}$. These different categories of trials were intermixed in a random order.

\section{Subjects}

The two authors served as subjects. They had normal (D.L.) and corrected-to-normal (D.W.) vision. All sessions were conducted at the same time of day, and, to anticipate the results, there were no significant differences of any kind between sessions.

\section{RESULTS}

Each subset of the data consists of a $2 \times 6$ matrix of frequencies-two stimulus orders $\times$ six response categories. Individual subsets of the data conform well to Tanner and Swets's (1954) normal, equal-variance, signal detection model, and we report $d^{\prime}$ as a statistically sufficient index of discrimination under each condition of interest. The squares in Figure 1 show how $d^{\prime}$ varied with ISI.

To exhibit the relation between $d^{\prime}$ and ISI over as wide a geometric range of retention intervals as possible, we also present in Figure 1 the data from Laming and Scheiwiller's (1985) Experiment 1. Laming and Scheiwiller measured a threshold for the discrimination of horizontal lines presented for $500 \mathrm{msec}$, and we have converted those thresholds (right-hand scale) to equivalent $d^{\prime}$ per $4.75 \%$ increment in length to align the data from Subject D.L., who served in both those experiments. This extends the range of temporal intervals to $64 \mathrm{sec}$. To characterize the trend in the data, we have fitted the empirical function

$$
d^{\prime}=3.0 /[\ln (\tau+1)+1.40],
$$

where the ISI $\tau$ is measured in seconds, to D.L.'s combined data. This function captures the logarithmic character of much data on retention (Laming \& Scheiwiller, 1985; 
Woodworth \& Schlosberg, 1955, pp. 724-728) and has the advantage over a simple linear function of the logarithm that it tends to a finite limit, as $\tau$ tends to zero, and to zero, as $\tau$ tends to infinity. It is very nearly the formula suggested by Ebbinghaus (1885/1964, pp. 77-78).

\section{DISCUSSION}

Following Sperling's (1960) pioneering work, most research on memory over very short intervals has used his partial-report technique with character stimuli. Recently Palmer $(1988,1990)$ has shown the partialreport technique to work equally well with noncharacter stimuli, including the discrimination of length of line. But that experimental procedure, with multiple stimuli presented on each trial, is more complicated than the much older technique we have used here. Analysis of partial-report data rarely extends to a quantitative model. Because our technique lends itself more readily to quantitative analysis, we see a useful role for it in elucidating the nature of retention over very short intervals.

We believe our present data to be representative of the retention of simple stimuli over very short intervals, uncompromised by cues arising from the configuration of the stimuli and extraneous interference from transfer of fixation and masking. But, in fact, there is no way in which one can show that this or any other experimental condition reveals a "pure" memory function except the circumstantial way of fitting a simple and plausible model to the data. Intuitively, we assume that the contribution from memory ought to decrease monotonically with lapse of time, so experiments showing a reversal of this trend (e.g., Laming \& Scheiwiller, 1985, Experiment 2) must, by that criterion, be compromised.

Further exploration of the relation between discriminability and ISI must await a larger study encompassing a sufficiently wide geometric range of intervals within one experiment. The purpose of this comment is to suggest how that experiment might be realized and to identify the kind of empirical trend one should expect and some of the hazards that might distort it.

\section{REFERENCES}

Burbeck, C. A. (1987). Position and spatial frequency in large-scale localization judgments. Vision Research, 27, 417-427.

Ebbinghaus, H. (1964). Memory (H. A. Ruger \& C. E. Bussenius, Trans.). New York: Dover. (Original work published 1885)

LAMiNG, D., SCheiwiller, P. (1985). Retention in perceptual memory: A review of models and data. Perception \& Psychophysics, 37, 189-197.

PAlmer, J. (1988). Very short-term visual memory for size and shape. Perception \& Psychophysics, 43, 278-286.

Palmer, J. (1990). Attentional limits on the perception and memory of visual information. Journal of Experimental Psychology: Human Perception \& Performance, 16, 332-350.

Sorkin, R. D. (1966). Temporal interference effects in auditory amplitude discrimination. Perception \& Psychophysics, 1, 55-58.

SPERLING, G. (1960). The information available in brief visual presentations. Psychological Monographs, 74(No. 498).

Swets, J. A., TANner, W. P., JR., Birdsall, T. G. (1961). Decision processes in perception. Psychological Review, 68, 301-340.

TANNER, W. P., JR. (1961). Physiological implications of psychophysical data. Annals of the New York Academy of Sciences, 89, 752-765.

TANNER, W. P., JR., \& SWETs, J. A. (1954). A decision-making theory of visual detection. Psychological Review, 61, 401-409.

TAYLOR, M. M., \& SMTH, S. M. (1975). Monaural detection with contralateral cue. V. Interstimulus interval in MDCC and amplitude discrimination. Journal of the Acoustical Society of America, 57, 1500-1511.

WOODWORTh, R. S., SCHLOSBERG, H. (1955). Experimental psychology (3rd ed.). London: Methuen.

(Manuscript received October 3, 1991.) 